\title{
Influence of combined oral contraceptives on the periodontal condition
}

\author{
Roberta Santos DOMINGUES ${ }^{1}$, Bruna Fidêncio Rahal FERRAZ1', Sebastião Luiz Aguiar GREGHI², Maria Lúcia Rubo \\ de REZENDE², Euloir PASSANEZI ${ }^{3}$, Adriana Campos Passanezi SANT'ANA²
}

1- DDS, MS, Division of Periodontology, Department of Prosthodontics, Bauru School of Dentistry, University of São Paulo, Bauru, SP, Brazil.
2- DDS, PhD, Associate Professor, Division of Periodontology, Department of Prosthodontics, Bauru School of Dentistry, University of São Paulo, Bauru, SP, Brazil.
3- DDS, PhD, Professor and Chair, Division of Periodontology, Department of Prosthodontics, Bauru School of Dentistry, University of São Paulo, Bauru, SP, Brazil.

Corresponding address: Adriana Campos Passanezi Sant'Ana - Disciplina de Periodontia - Departamento de Prótese - Faculdade de Odontologia de Bauru - Universidade de São Paulo (FOB/USP) - Al. Octávio Pinheiro Brisolla, 9-75 - - 17012-901 - Bauru - SP - Brazil - Phone +55 (14) $3235-8366$ - Fax + (14) 3235-8245 - e-mail: drisantana@yahoo.com.br

Received: September 19, 2010 - Modification: May 03, 2011 - Accepted: June 01, 2011

\section{ABSTRACT}

Most studies investigating the impact of oral contraceptives have been performed some years ago, when the level of sexual hormones was greater than the actual formulations. Objective: The aim of this study was to evaluate the effects of current combined oral contraceptives (COC) on periodontal tissues, correlating the clinical parameters examined with the total duration of continuous oral contraceptive intake. Material and methods: Twenty-five women (19-35 years old) taking combined oral contraceptives for at least 1 year were included in the test group. The control group was composed by 25 patients at the same age range reporting no use of hormone-based contraceptive methods. Clinical parameters investigated included pocket probing depth (PD), clinical attachment level (CAL), sulcular bleeding index (SBI) and plaque index (PI.I). Data were statistically evaluated by unpaired t test, Pearson's correlation test and Spearman's correlation test. Results: The test group showed increased PD $(2.228 \pm 0.011 \times 2.154 \pm 0.012 ; p<0.0001)$ and SBI $(0.229 \pm 0.006 \times 0.148 \pm 0.005, p<0.0001)$ than controls. No significant differences between groups were found in CAL $(0.435 \pm 0.01 \times 0.412 \pm 0.01 ; p=0.11)$. The control group showed greater PI.I than the test group $(0.206 \pm 0.007 \times 0.303 \pm 0.008 ; p<0.0001)$. No correlation between the duration of oral contraceptive intake, age and periodontal parameters was observed. Conclusions: These findings suggest that the use of currently available combined oral contraceptives can influence the periodontal conditions of the patients, independently of the level of plaque accumulation or total duration of medication intake, resulting in increased gingival inflammation.

Key words: Oral contraceptives. Estrogens. Progesterone. Gingivitis. Periodontitis.

\section{INTRODUCTION}

Gingivitis associated with hormonal changes, such as those observed in puberty and pregnancy, seems to be dependent on, but unrelated to, the amount of plaque accumulation ${ }^{3}$. The main premenopausal steroid sex hormones (estradiol and progesterone) are responsible not only for the physiological changes in women at different phases of their lifetime, but also for significant biological actions that can affect different organ systems including the oral cavity ${ }^{15,21}$.

Clinically, a positive correlation between estradiol and progesterone serum levels and gingival inflammation, regardless the amount of plaque accumulation, has been described in puberty-associated gingivitis ${ }^{15,17}$. It has also been suggested that the exacerbated inflammatory response to irritant factors could be explained by qualitative changes in microbiota $2,11,17,21$ or host response to plaque accumulation $2,4,9,10,14,21$. Some bacterial species possess the ability to use menadione as a substitute for vitamin $\mathrm{K}$, leading to an explosion of its levels at subgingival environment, resulting in the clinical presentation of gingival inflammation ${ }^{2,11,17,21}$. Also, increased sex hormone levels in serum could induce tissue destruction by activating matrix metalloproteinases 
proteins (MMPs) ${ }^{12}$.

The use of hormonal contraceptives by women at their reproductive life has been considered to influence periodontal disease progression ${ }^{2,5,15,16,21}$. Clinical studies reported higher prevalence of gingival inflammation, loss of attachment and gingival enlargement in woman taking hormonebased oral contraceptives $8,10,19,21$. However, recent studies based on large ${ }^{23}$ or small ${ }^{20}$ population samples suggested that current combined oral contraceptives (COC) do not affect periodontal health, possibly related to their lower concentration of progesterone and estradiol than previously used medications ${ }^{16}$. Gingival inflammation seems to be associated to high concentrations of sex hormones present in $\mathrm{COC}^{19}$. It is also noteworthy that an increased gingival inflammation are dependent on the time of use of $\mathrm{COC}^{10,19}$.

Since most of the studies reporting the influence of oral contraceptives on periodontal tissues were published during the 1960's and 1970's, when hormonal doses of contraceptives were higher than those of current formulations, the aim of this study was to investigate the effects of current low-dose $\mathrm{COC}$ on clinical periodontal conditions of healthy young adult women continuously taking such medications for at least 1 year.

\section{MATERIAL AND METHODS}

\section{Sample selection}

Ethical approval was obtained from the Bauru School of Dentistry Ethical Board prior to commencement and written informed consent was obtained from all subjects prior to enrollment. Were invited to participate in this study 50 women aged 19-35 years old, systemically health, presenting at least 24 teeth (except for third molars). To be included in the test group $(n=25)$ women should report continuous use of COC (progesterone+estradiol) for at least 1 year. Women included in the control group $(n=25)$ should report no use of COC.

Exclusion criteria were: current pregnancy or delivery within 12 months prior to data collection; smoking or former smokers; drugs or alcohol abuse; mouth breathing; history of radiotherapy or chemotherapy in the past 5 years; early or premature menopause; breastfeeding; presence of any systemic condition that could influence host response to plaque accumulation (e.g.: diabetes mellitus); use of antibiotics, non-steroidal or steroidal anti-inflammatory drugs for the previous 6 months; periodontal treatment, including professional prophylaxis, within the 6-month period prior to data collection; use of drugs that could induce gingival enlargement (e.g.: calcium channel blockers, cyclosporine or anti-convulsants); interrupted use of $\mathrm{COC}$; and hormone replacement therapy.

Volunteers were selected among dental undergraduate and graduate students at Bauru School of Dentistry, University of São Paulo, Brazil, between August and November 2008. For each patient included in the test group, a volunteer at the same or similar age not reporting the use of COC and not presenting any of the exclusion criteria was selected to compose the control group, in such a way that the test and control groups were composed by 25 age-matched women each.

A detailed questionnaire was completed by all patients included in the study, recording full medical history and $\mathrm{COC}$ intake before clinical examination. Test group volunteers were questioned about the name, dose and total uninterrupted use of combined oral contraceptives, which were recorded in patient file record chart.

\section{Periodontal examination}

Patients were evaluated by a previously calibrated ( $K=0.92$ for probing depth and $K=0.89$ for clinical attachment level), single blinded examiner according to pocket probing depth (PD), gingival recession (REC) or hyperplasia (HP), clinical attachment level (CAL), sulcular bleeding index (SBI) and plaque index (PI.I). All patients were examined using good illumination and standardized conditions. Full-mouth examination was performed at six sites per tooth for PD, REC, HP, CAL and SBI and at four sites per tooth for PI.I, except for third molars.

PD - measured with a $15-\mathrm{mm}$ conventional periodontal probe (Hu-Friedy, Chicago, IL, USA), determined by the distance from gingival margin to the bottom of the gingival sulcus or pocket;

REC and HP - measured with a 15-mm conventional periodontal probe (Hu-Friedy) as the distance from gingival margin to cementoenamel junction (CEJ). REC was recorded at patients file record as a negative value and HP was recorded as a positive value, in order to allow the determination of CAL;

CAL - defined as the distance from CEJ to the bottom of the sulcus or pocket, derived from the measures of PD and REC or HP, as exemplified by the formula ${ }^{27}$ :

$C A L=P D-[R E C$ or $H P] C A L=P D-[R E C$ or HP $]$

PI.I - The presence or absence of plaque was determined following the proposal of O'Leary, Drake and Naylor ${ }^{18}$ (1972). According to that, the presence of plaque was recorded as 1 and its absence as 0;

SBI - the occurrence of bleeding on gentle probing was observed within $15 \mathrm{~s}$ after removal of the probe from the sulcus and recorded as present (1) or absent (0), as proposed by Ainamo \& Bay ${ }^{1}$ (1975). 


\section{Statistical analysis}

Data were analyzed using the GraphPad Prism 5 statistical software (GraphPad Software Inc., San Diego, CA, USA). Determination of sample size was based on other studies reporting the investigation of $\mathrm{COC}$ influence on periodontal status ${ }^{16,20}$. For statistical purposes, the unit of analysis was the site. The comparison of clinical periodontal parameters (PD, CAL, SBI and PI.I) between test and control groups was performed by unpaired $t$ test at a significance level of $5 \%(\alpha=0.05)$. The correlation between the total duration of COC intake, age and clinical periodontal parameters was investigated by Pearson's correlation test for linear measurements (PD and CAL) and by Spearman's correlation test for non-linear measurements (SBI and PI.I) at a significance level of 5\%.

\section{RESULTS}

The mean age for test and control groups did not differ significantly $(24.12 \pm 0.371 \times 25.92 \pm 0.978$ years; $\mathrm{p}>0.05$; unpaired $t$ test). The mean duration of COC intake for the test group was $4.34 \pm 2.49$ years. Among the various $\mathrm{COC}$ formulations used by the subjects, estrogen content ranged from 0.015 to $0.04 \mathrm{mg}$ and progestin content ranged from 0.075 to $3.0 \mathrm{mg}$ (Figure 1 ). The mean duration of COC intake in the test group was $4.44 \pm 2.42$ years.

Clinical periodontal parameters of patients included in test and control groups are shown at Table 1. Full-mouth evaluation provided the analysis of 4,116 sites for PD, CAL and SBI and 2,735 sites for PI.I at control group, and 4,134 sites for PD, CAL and SBI and 2,757 sites for PI.I for the test group. Comparative analysis by unpaired $t$ test showed that test group patients presented greater PD and SBI than controls $(p<0.0001)$. No significant differences in CAL were found between groups $(p=0.11)$. Controls showed increased PI.I than test group $(p<0.0001)$.

The analysis by Pearson's and Spearman's correlation test in the test group (Table 2) showed no statistically significant correlation between the total duration of COC intake or age and clinical periodontal parameters $(p>0.05)$. A significant

Figure 1- Concentration of sex hormones (progestin and estradiol) of the different brands of combined oral contraceptives consumed by patients in the test group

\begin{tabular}{|c|c|c|}
\hline Brand \# & Progestin and estradiol concentration & Number of patients \\
\hline 1 & $3 \mathrm{mg}$ drospirenone; $0.02 \mathrm{mg}$ ethynil estradiol & 5 \\
\hline 2 & $0.60 \mathrm{mg}$ gestoden; $0.015 \mathrm{mg}$ ethynil estradiol & 1 \\
\hline 3 & $3 \mathrm{mg}$ drospirenone; $0.03 \mathrm{mg}$ ethynil estradiol & 1 \\
\hline 4 & $0.075 \mathrm{mg}$ gestoden; $0.03 \mathrm{mg}$ ethynil estradiol & 2 \\
\hline 5 & $2 \mathrm{mg}$ cyproterone; $0.035 \mathrm{mg}$ ethynil estradiol & 5 \\
\hline 6 & $0.075 \mathrm{mg}$ gestoden; $0.2 \mathrm{mg}$ ethynil estradiol & 2 \\
\hline 7 & 7 pills of $0.25 \mathrm{mg}$ desogestrol and $0.04 \mathrm{mg}$ ethynil estradiol; 14 pills of $0.125 \mathrm{mg}$ & 1 \\
\hline 8 & $2 \mathrm{mg}$ cyproterone; $0.035 \mathrm{mg}$ ethynil estradiol & 2 \\
\hline 9 & $2 \mathrm{mg}$ acetate clormadiome; 0.03 mg ethynil estradiol \\
\hline 10 & $0.075 \mathrm{mg}$ gestoden; $0.02 \mathrm{mg}$ ethynil estradiol & \\
\hline 11 & $0.15 \mathrm{mg}$ desogestrol; $0.02 \mathrm{mg}$ ethynil estradiol & \\
\hline
\end{tabular}

Table 1- Clinical periodontal parameters in the test and control groups (unpaired $t$ test)

\begin{tabular}{cccccccc}
\hline & & Test & & & Control & & P value \\
& $\mathbf{n}$ & Mean & SD & $\mathbf{n}$ & Mean & SD & \\
\hline PD & 4134 & 2.228 & 0.011 & 4116 & 2.154 & 0.012 & $\mathrm{p}<0.0001^{*}$ \\
CAL & 4134 & 0.435 & 0.010 & 4116 & 0.412 & 0.010 & $\mathrm{p}=0.1184$ \\
SBI & 4134 & 0.229 & 0.006 & 4116 & 0.148 & 0.005 & $\mathrm{p}<0.0001^{*}$ \\
PI.I & 2757 & 0.206 & 0.007 & 2735 & 0.303 & 0.008 & $\mathrm{p}<0.0001^{*}$ \\
\hline
\end{tabular}

PD- probing depth (in millimeters); CAL-clinical attachment level (in millimeters); SBI- sulcus bleeding index (0-absence; 1- presence); PI.I- plaque index (0- absence; 1- presence); $n=$ number of sites evaluated; SD- standard deviation; *significant if $p<0.05$ 
Table 2- Correlation between age, total duration of oral contraceptive therapy and clinical periodontal parameters in test group

\begin{tabular}{|c|c|c|c|c|c|}
\hline & Duration of use $e^{*}$ & PD* & $\mathrm{CAL}^{*}$ & SBI $I^{* *}$ & PI. I** \\
\hline \multirow[t]{2}{*}{ Age } & $r=0.471$ & $r=-0.252$ & $r=-0.366$ & $r=-0.200$ & $\begin{array}{l}-- \\
--\end{array}$ \\
\hline & $p=0.018 \dagger$ & $p=0.225$ & $p=0.071$ & $p=0.338$ & \\
\hline \multirow[t]{2}{*}{ Duration of use } & --- & $r=-0.133$ & $r=-0.095$ & $r=0.039$ & --- \\
\hline & & $p=0.652$ & $p=0.652$ & $p=0.673$ & \\
\hline \multirow[t]{2}{*}{ PD } & $r=-0.133$ & --- & $r=0.392$ & $r=-0.005$ & $r=0.005$ \\
\hline & $p=0.527$ & & $p<0.0001 \dagger$ & $p=0.747$ & $p=0.800$ \\
\hline \multirow[t]{2}{*}{$\mathrm{CAL}$} & $r=-0.095$ & $r=0.392$ & --- & $r=0.057$ & $r=-0.017$ \\
\hline & $p=0.675$ & $p<0.00001 \dagger$ & & $p=0.00007 \dagger$ & $p=0.381$ \\
\hline \multirow[t]{2}{*}{ SBI } & $r=0.088$ & $r=-0.001$ & $r=0.067$ & --- & $r=-0.060$ \\
\hline & $p=0.527$ & $p=0.930$ & $p=0.000002 \dagger$ & & $p=0.002 \dagger$ \\
\hline
\end{tabular}

PD- probing depth (in millimeters); CAL- clinical attachment level (in millimeters); SBI- sulcus bleeding index (0 absence; 1 - presence); PI.I.- plaque index ( 0 - absence; 1 - presence); $r=$ correlation coeficient; $p=p$ value, $\dagger$ significant if $<0.05$; Pearson's correlation test; ${ }^{* *}$ Spearman's correlation test

Table 3- Correlation between age and clinical periodontal parameters in the control group

\begin{tabular}{|c|c|c|c|c|}
\hline & PD* & $\mathrm{CAL}^{*}$ & SBI $I^{* *}$ & PI.I** \\
\hline \multirow[t]{2}{*}{ Age } & $r=0.014$ & $r=0.493$ & $r=0.161$ & $r=0.000$ \\
\hline & $p=0.948$ & $p=0.012 \dagger$ & $p=0.443$ & $p=1.000$ \\
\hline \multirow[t]{2}{*}{ PD } & -- & $r=0.026$ & $r=-0.006$ & $r=0.089$ \\
\hline & & $p=0.105$ & $p=0.699$ & $p<0.0001 \dagger$ \\
\hline \multirow[t]{2}{*}{ CAL } & $r=0.026$ & --- & $r=0.140$ & $r=0.052$ \\
\hline & $p=0.106$ & & $p<0.0001 \dagger$ & $p=0.007 \dagger$ \\
\hline \multirow[t]{2}{*}{ SBI } & $r=-0.006$ & $r=0.117$ & --- & $r=0.008$ \\
\hline & $p=0.724$ & $p<0.0001 \dagger$ & & $p=0.669$ \\
\hline
\end{tabular}

PD- probing depth (in millimeters); CAL- clinical attachment level (in millimeters); SBI- sulcus bleeding index (0 absence; 1 - presence); PI.I- plaque index (0 - absence; 1 - presence); $r=$ correlation coeficient; $p=p$ value, †significant if $<0.05$; ${ }^{*}$ Pearson's correlation test; ${ }^{* *}$ Spearman's correlation test

positive correlation was observed between CAL and PD, SBI and PI.I and CAL and SBI $(p<0.05)$.

The analysis by Pearson's and Spearman's correlation tests in the control group (Table 3 ) showed a positive correlation between age and CAL, PD and PI.I, CAL and SBI, and CAL and PI.I.

\section{DISCUSSION}

This study investigated the influence of current low-dose COC on clinical periodontal parameters of systemically healthy non-smoking women, and the correlation among these parameters, duration of usage and age. Our findings suggest that current COC therapy results in greater PD and SBI, regardless of the amount of plaque accumulation or duration of contraceptive intake.

Recently, Haerian-Ardakaniet, et al. ${ }^{6}$ (2010) evaluated the influence of current oral contraceptive pills on periodontal health in young women, aged 17 to 35 years, as evaluated in our study. Patients have completed a questionnaire to assess current and previous oral contraceptive use. Periodontal examination included PI.I, SBI, PD and CAL at six sites per tooth, as also performed in the present study. A total of 35 women taking oral contraceptives for at least 2 years were compared with 35 women not taking the medication, matched by age range and socioeconomic, occupational and educational levels. The results obtained showed that, although no difference in PI.I was observed, patients taking contraceptive pills had higher gingival inflammation and bleeding on probing. No significant differences were found between groups with respect to PD and CAL. Similar findings were previously described by Kalkwarf, et al.7 (1978), who suggested that women taking oral contraceptives had more gingival inflammation and lesser PI.I than controls not taking 
the medications, as shown in the present study. No differences were found among patients taking the different medications for 1-11 months, 12-35 months or $\geq 36$ months, independently from the medication brands.

The fundamental principles of COC are based on the use of hormones to simulate a pregnancy state to prevent ovulation to occur. Some previous study have reported that physiological hormonal imbalances occurring during puberty, pregnancy or menstrual cycle are associated with gingival inflammation, mainly related to deeper PD measures, gingival enlargement and bleeding on probing $2,12,13,15,19,25$. The incidence of gingival inflammation during puberty or pregnancy seems to be positively correlated with plasma steroid hormone concentration and independent from dental plaque scores ${ }^{15}$.

Our findings showed an greater PD and bleeding on probing in women taking $\mathrm{COC}$ for at least 1 year, which is agreement with other reports in literature ${ }^{6,12,15,16,21}$. Tilakaratne, et al. ${ }^{24}$ (2000) investigated 32 women taking hormonal contraceptives for less than 2 years, 17 of them for 2-4 years and a matched control group of 39 women not taking the medication. The results obtained suggested that women taking pills had similar oral hygiene levels than women who did not take. However, a significant higher level of gingival inflammation was observed in women taking pills. Also, their findings showed that women who took pills for 2-4 years had higher CAL than controls, which was not observed in the present study, since no differences in CAL was observed between test and control groups.

These findings suggest that the increase in PD are related to gingival hyperplasia or "false pocketing", rather than to destruction of supporting periodontal tissues ${ }^{2,12,16,21}$, similarly to those observed during pregnancy or puberty $2,15,21$. However, these findings were not observed in other studies ${ }^{9,14,28}$. It has been discussed that, although some qualitative alterations occur in the subgingival bacterial microbiota influenced by sex hormones, women capable of maintaining efficient oral hygiene habits do not present alterations on clinical parameters, such as gingival index, PI.I and PD ${ }^{9}$. However, considering the disturbances in microvascular structure and functions, vasodilatation, increased permeability, and the increase in crevicular fluid flow triggered by the presence of higher levels of progesterone and estrogen, the presence of greater PD in the test group could be explained by the fact that these alterations exacerbate the inflammatory response and might result in gingival hyperplasia ${ }^{13,15,22}$.

As discussed by Kalkwarf, et al.7 (1978), the increased inflammatory response observed in patients taking oral contraceptives is probably due to the effects of artificially elevated levels of progesterone, which has been shown to alter the microvascular topography and permeability of gingival tissues, increase the number of PMN within gingival sulcus and, in combination with estradiol, increase the synthesis of $\mathrm{PGE}_{2}$ in gingival tissues. According to them, each of these mechanisms could be responsible for causing an exaggerated gingival response to local irritants. As their study was conducted in 1978, when the concentrations of sex hormones in the medications formulations were higher than those existing currently, it seemed reasonable that these negative adverse effects could be overcome. However, the findings of the present study indicate that the current COC formulations do influence periodontal health, since more bleeding on probing and greater PD were observed in test than control groups.

Such result might be due to the possible absence of receptors to these sex hormones on the periodontal tissues, suggesting that any pharmacological effects exerted by estrogen and progesterone on this tissue are indirect ${ }^{9}$. Another possible explanation for such finding would be that current COC present extremely low dosage of estrogen and progesterone, not contributing significantly to periodontal disease progression, especially in women that maintain optimal hygiene habits $^{15,18}$. However, it should be emphasized that there are reports of the presence of estrogen and progesterone receptors in the periodontal tissues ${ }^{25,26}$ making alterations on cytokine and prostaglandin production in association with disturbances in the microvascular system and higher levels of certain bacterial species, as a result of the effects of higher serum levels of sex hormones during the use of oral contraceptives. These arguments are supportive to the belief that women taking this medicine are more susceptible to the development and progression of periodontal diseases $2,10,11,14,16,24$.

In the methodological design of the study, special care was taken to control other variables that could influence the development of gingival enlargement or periodontal destruction, including smoking habits, diabetes, previous periodontal treatment, use of specific medications, among others. Therefore, the influence of COC could be fairly investigated in this study. Patients taking COC showed significantly lesser amount of plaque accumulation than controls, though showing higher SBI and greater PD. These findings are in agreement with other reports in literature that suggested that the degree of gingival inflammation in patients with physiological or pathological imbalances in sex hormones serum levels is not directly correlated to the amount of plaque accumulation 2,7,9-11,14,21,28. In addition, no correlation between PI.I and PD or CAL 
and a weak but significant negative correlation with SBI were observed in the test group, reinforcing these results.

This exacerbated host response could be credited to a qualitative change in subgingival microbiota influence by an increase in sex hormone levels, as suggested before ${ }^{11,14,17,19}$, or to an alteration of host response to plaque accumulation ${ }^{2,12,15,21}$. Some studies suggested that the elevation in sex hormone levels result in an increased synthesis of cytokines and prostaglandins that are intimately associated with tissue destruction and bone resorption, by activating matrix metalloproteinases and proteolytic enzymes as well as by osteoclast formation ${ }^{12}$.

Brusca, et al. ${ }^{5}$ (2010), after investigating the influence of oral contraceptives use on the subgingival occurrence of specific periodontopathogens and the host's periodontal status in 92 women aged 19-40 years, showed that women taking oral contraceptives showed a significant increase in the prevalence of severe periodontitis, specially smokers. Also, these women had higher numbers of cultures positive for Candida $s p$. , which were associated with $P$. gingivalis and $P$. intermedia.

It is known that not only the prevalence, but also the periodontal disease severity increases with age due to a prolonged exposure to numerous risk factors throughout life. This study did not obtain a positive correlation between PI.I, SBI and PD and age, but there was statistically significant positive correlation between $\mathrm{CAL}$ and age in the control group. Other authors have reported positive correlation between age and $\mathrm{PD}^{17,28}$, and between age and loss of attachment ${ }^{21}$.

The hormonal dosage and the total duration of oral contraceptive intake are two possible factors influencing the effects of this medicine on the periodontal condition. Considering the increased production of pro-inflammatory cytokines and prostaglandins resultant from elevated levels of these hormones, it seems reasonable to suggest that a continued exposure for a long period of oral contraceptive use results in a higher risk to periodontal disease development and progression $7,9,10,12,14$. In this study, there was no statistically significant positive correlation between the clinical parameters analyzed the total duration of oral contraceptive intake. These findings can be explained by the low hormonal dosage of the current oral contraceptive formulations used by the patients selected in this study, in agreement with other results obtained with women taking low-dose oral contraceptives, which found no statistically significant differences in the clinical parameters $\mathrm{s}^{20,23}$. However these results are contradictory as it has been observed that women taking COC with low hormonal dosage present greater periodontal inflammation, greater PD and loss of attachment than women not taking these medications ${ }^{16}$. In addition, Knight and Wade ${ }^{10}$ (1974) found that subjects who had been on oral contraceptive therapy for longer than 1.5 years showed increased attachment loss than those taking contraceptives for a shorter period of time or those who did not report oral contraceptive intake.

Even though the influence of oral contraceptives on the periodontal condition has been evaluated, several mechanisms involved in the process are yet to be explained13,19,21,22. Apart from that, there are still a small number of studies analyzing the effects of current COC, which present a reduced hormonal dosage in comparison with the oral contraceptives evaluated by most of the studies published on this subject. Therefore, the effects of these medications in the development and progression of gingival inflammation are not completely known. In order to elucidate and complement the results obtained here, additional studies must be developed examining the effects of current COC intake for different periods of time to determine their influence on the periodontal tissues.

\section{CONCLUSIONS}

In summary, currently available COC might influence the periodontal condition of women taking these medications for at least 12 months continuously, regardless of age and amount of plaque accumulation, resulting in increased PD and SBI and a slight tendency to develop loss of attachment.

\section{REFERENCES}

1- Ainamo J, Bay I. Problems and proposals for recording gingivitis and plaque. Int Dent J. 1975;25:229-35

2- Amar S, Chung KM. Influence of hormonal variation on the periodontium in women. Periodontology 2000. 1994;6:79-87.

3- Armitage GC. Development of a classification system for periodontal diseases and conditions. Ann Periodontol. 1999;4:1-6. 4- Beck JD, Offenbacher S. Relationships among clinical measures of periodontal disease and their associations with systemic markers. Ann Periodontol. 2002;7:79-89.

5- Brusca MI, Rosa A, Albaina O, Moragues MD, Verdugo F, Pontón J. The impact of oral contraceptives on women's periodontal health and subgingival occurrence of aggressive periodontopathogens and Candida species. J Periodontol. 2010;81:1010-8.

6- Haerian-Ardakani A, Moeintaghavi A, Talebi-Ardakani MR, Sohrabi K, Bahmani S, Dargahi M. The association between current low-dose oral contraceptive pills and periodontal health: a matched-case-control study. J Contemp Dent Pract. 2010;11:3340.

7- Kalkwarf KL. Effect of oral contraceptive therapy on gingival inflammation in humans. J Periodontol. 1978;49:560-3.

8- Kaufman AY. An oral contraceptive as an etiologic factor in producing hyperplastic gingivitis and a neoplasm of the pregnancy tumor type. Oral Surg Oral Med Oral Pathol. 1969;28:666-70.

9- Klinger G, Eick S, Klinger G, Pfister W, Gräser T, Moore C, et al. Influence of hormonal contraceptives on microbial flora of gingival sulcus. Contraception. 1998;57:381-4. 
10- Knight GM, Wade AB. The effects of hormonal contraceptives on the human periodontium. J Periodontal Res. 1974;9:18-22.

11- Kornman KS, Loesche WJ. Effects of estradiol and progesterone on Bacteroides melaninogenicus and Bacteroides gingivalis. Infect Immun. 1982;35:256-63.

12- Lapp CA, Lapp DF. Analysis of interleukin-activated human gingival fibroblasts: modulation of chemokine responses by female hormones. J Periodontol. 2005;76:803-12.

13- Lynn BD. "The pill" as an etiologic agent in hypertrophic gingivitis. Oral Surg Oral Med Oral Pathol. 1967;24:333-4.

14- Machtei EE, Mahler D, Sanduri H, Peled M. The effect of menstrual cycle on periodontal health. J Periodontol. 2004;75:40812.

15- Mariotti A. Sex steroid hormones and cell dynamics in the periodontium. Crit Rev Oral Biol Med. 1994;5:27-53.

16- Mullally BH, Coulter WA, Hutchinson JD, Clarke HA. Current oral contraceptive status and periodontitis in young adults. J Periodontol. 2007;78:1031-6.

17- Nakagawa S, Fuji H, Machida Y, Okuda K. A longitudinal study from prepuberty to puberty of gingivitis. Correlation between the occurrence of Prevotella intermedia and sex hormones. J Clin Periodontol. 1994;21:658-65.

18- O'Leary TJ, Drake RB, Naylor JE. The plaque control record. J Periodontol. 1972;43:38.

19- Pankhurst CL, Waite IM, Hicks KA, Allen Y, Harkness RD. The influence of oral contraceptive therapy on the periodontium duration of drug therapy. J Periodontol. 1981;52:617-20.
20- Preshaw PM, Knutsen MA, Mariotti A. Experimental gingivitis in women using oral contraceptives. J Dent Res. 2001;80:2011-5. 21- Sooriyamoorthy M, Gower DB. Hormonal influences on gingival tissue: relationship to periodontal disease. J Clin Periodontol. 1989;16:201-8.

22- Sperber GH. Oral contraceptive hypertrophic gingivitis. J Dent Assoc S Afr. 1969;24:37-40.

23- Taichman LS, Eklund SA. Oral contraceptives and periodontal diseases: rethinking the association based upon analysis of National Health and Nutrition Examination Survey data. J Periodontol. 2005;76:1374-85.

24- Tilakaratne A, Soory M, Ranasinghe AW, Corea SMX, Ekanayake SL, Silva M. Effects of hormonal contraceptives on the periodontium, in a population of rural Sri-Lankan women. J Clin Periodontol. 2000;27:753-7.

25- Vittek J, Gordon GG, Rappaport SC, Munnangi PR, Southren AL. Specific progesterone receptors in rabbit gingiva. J Periodontal Res. 1982;17:657-61.

26- Vittek J, Hernandez MR, Wenk EJ, Rappaport SC, Southren AL. Specific estrogen receptors in human gingiva. J Clin Endocrinol Metabol. 1982;54:608-12.

27- Wood S, Frydman A, Cox S, Brant R, Needoba S, Eley B, et al. Periodontal disease and spontaneous preterm birth: a case control study. BMC Pregnancy Childbirth. 2006;6:24.

28- Yalcin F, Basegmez C, Isik G, Berber L, Eskinazi E, Soydinc $M$, et al. The effects of periodontal therapy on intracrevicular prostaglandin E2 concentrations and clinical parameters in pregnancy. J Periodontol. 2002;73:173-7. 